\title{
Heat Extraction Performance of EGS with Heterogeneous Reservoir: a Numerical Evaluation
}

\author{
Wenbo Huang Wenjiong Cao Fangming Jiang*
}




\begin{abstract}
Strong heterogeneity does exist in engineered heat reservoirs of enhanced geothermal systems (EGSs) due to different rock formation, pre-existence of natural fractures and uncertainties associated with the stimulation processes. Exploring the influence of reservoir heterogeneity on EGS heat extraction is of considerable importance to predict and fully evaluate the performance of EGS. In this work, we take the reservoir as equivalent to a porous medium and employ a previously self-developed numerical model to conduct a series of simulations of the long-term heat extraction process of EGSs with different reservoir porosity distributions. Based on a detailed analysis to the effects of fluid seepage flow field on the heat extraction performance, we establish a method to quantify the heat extraction performance of the EGS with heterogeneous reservoir using the seepage distribution data. We numerically test this method by taking a wide variety of heterogeneous reservoirs for the EGSs. The method proves to have the capability of predicting the heat extraction performance of EGSs with miscellaneous heterogeneous reservoirs.
\end{abstract}

Keywords: enhanced geothermal system (EGS); numerical simulation; equivalent porous medium; heterogeneity; heat extraction. 


\section{Introduction}

\subsection{Background of Enhanced Geothermal System}

The core of Earth has a temperature well over $4000 \mathrm{~K}$ [1] resulting in an enormous amount geothermal energy stored underground. The World Energy Assessment (WEA) estimates that the geothermal energy within the upper 5-kilometer shell of the earth is about $140 \times 10^{6}$ EJ [2], which is practically an infinite resource considering that the present annual global energy consumption is just 570 EJ [3]. Geothermal energy has the potential of fulfilling the base-load electric power requirements with little or no environmental footprint [4]. Exploiting geothermal energy might be an important strategy to meet the fast-growing energy demand, while reducing carbon emissions. At present, the installed geothermal power worldwide is only $0.38 \mathrm{EJ} /$ year (12,000 MW) [5], which represents a minor fraction of the available geothermal energy. Present exploitation of geothermal energy falls primarily into the hydrothermal resource category, in which natural fracture networks have fluid present and permit fluid circulation. The requirement of a particular type of geologic structures limits the hydrothermal electricity generation to a limited number of regions in the world.

Hot dry rock (HDR) energy is another form of geothermal energy, representing a vast store of thermal energy that is contained in the hot impervious crystalline basement rocks and can be found almost everywhere beneath the earth's surface within a depth of $3 \mathrm{~km}$ to $10 \mathrm{~km}$. Investigation shows more than $90 \%$ of the total US 
geothermal resource is stored in HDR [6]. Aiming to extract the energy stored in HDR, the prototype of an Enhanced Geothermal System (EGS) was designed and implemented by the Los Alamos Laboratory in 1970s. The initial concept is straightforward: for low-permeability rocks, a series of rock-fracturing procedures, such as hydraulic stimulation to create an artificially fractured reservoir is performed. By circulating water through the stimulated region, heat can be continuously extracted from the rock, just like a natural hydrothermal system. EGS has drawn a lot of attention over the past 40 years and a large amount of research and field testing has been carried out worldwide [7].The investigation report by an MIT-led interdisciplinary panel [8] assessed in detail the resource potential and technological feasibility of previous EGS projects and experiments and estimated the cost-competitive electricity generating capacity of EGS could reach $100 \mathrm{GW}$ in US within 50 years. Nonetheless, for the time being, EGS engineering still requires knowledge based on science and unified project experience to produce useful amounts of energy economically. There is an ample range of actions to tap this technology, and performance improvement of engineered reservoirs is one of the key issues $[7,8]$.

Creating a sufficiently large fractured rock volume is one of the primary requirements for reservoir stimulation [8]. Meanwhile, engineered reservoirs of EGSs serve not only as heat storage but also as heat exchanger; during the heat-mining operations, the production temperature would inevitably decline as a result of reservoir cooling-down. The economic effectiveness of EGS power generation is sensitive to the drop in production temperature [9]; therefore, the economic benefit 
derived from the exploitation of geothermal heat will be much smaller than expected from the total heat stored in the fracture volume if the reservoir has poor heat exchange effectiveness. Considering the high cost of well drilling and the relatively low-cost of reservoir stimulation, there will be major economic rewards by having a well-constructed reservoir, which will be capable of maintaining the production temperature at an adequate level through the EGS lifetime. Reliable tools for reservoir simulation may contribute decisively toward EGS economic feasibility.

\subsection{Reservoir structure and modeling}

When the EGS concept was initially proposed by Robinson et al. [10] of the Los Alamos Laboratory, the structure of engineered reservoirs was identified as essentially "penny-shaped" fractures connecting the injection and production wells, mainly based on the empirical hydraulic fracturing concepts of sedimentary rocks, which considered the induced fractures as planar and normal to the axis of the least principal stress [11]. Multiple parallel fracture structures were then suggested by Raleigh et al. [12] in which the geothermal wells were drilled at an angle in a direction perpendicular to the expected orientation of the fractures to create a series of parallel cracks from a single well. The concept associated to single and multiple fractures assumed that the host rock behaved as a continuum; however, it was later found not being appropriate for the crystalline rock in most HDR cases, where the pre-existing joints were pervasive and much weaker than the host matrix [13]. Further experiments dealing with field hydraulic fracturing indicated that the creation of new hydraulic 
fractures was not the dominant process; in fact, the shearing of natural joints and fractures favorably aligned with the principal directions of the local stress field was the prevailing process [14]. The existing joints and fractures would prevent the extension of artificial fractures; meanwhile, they could be sheared and widened during the stimulation process thus causing a fracture network with increased permeability [15]. Affected by the uneven distributed joints and faults in host rocks, the actual fractured HDR reservoir behaves like interconnected networks of flow paths with a few dominant routes acting as flow conduits [16].

The idealized structure of an EGS reservoir is a sufficiently fractured volume with large enough heat exchange area and without severe preferential flow paths. However, the stimulation of an EGS reservoir is a complicated process, which can be affected by many factors, such as the rock local stress state [13], natural fractures [13], and temperature variation [17], thus the development of fractures is rather difficult to control and predict. Brown [18] summarized the main experience obtained from the early EGS field research in which an HDR reservoir should be first created from the initial borehole, and then drilled the production borehole close to the elongated boundaries of the seismically determined reservoir region. Field and laboratory experiments have demonstrated strong evidence of channeling and highly preferential flow paths in individual fractures and in fracture networks. In EGS engineering, to further improve the performance of EGS system, the common strategy after creating an EGS reservoir also includes many subsequent amendments, such as re-drilling, secondary stimulations, and sealant treatments [19]. 
To guide the process of reservoir engineering, a thorough understanding of the relation between reservoir structure and EGS thermal performance is needed. Since EGS field experiments are demanding in terms of cost and time, analyses and simulation models seem to be the most effective means of predicting EGS long-term performance for a particular reservoir structure. In early years, constrained by the availability of adequate computing resources, the reservoir was commonly treated as a single or parallel planar fracture in order to obtain analytical solutions [20-23]. These analytical solutions were typically presented in some sort of line graphs of dimensionless temperature versus dimensionless time [20, 21], which can be converted to obtain the variation in production temperature and a quantitative estimate of the EGS performance. It should be noted that fracture spacing is of particular importance in the parallel fracture model. Gringarten et al. [21] presented an analytical solution of heat extraction from fractured hot dry rock with an infinite series of parallel vertical fractures of uniform aperture, and indicated that: 1) the multiple fracture system would provide a distinctly higher effectiveness at heat extraction than a single fracture system; and 2) the fracture spacing would have strong effects on the heat extraction performance (smaller is the spacing, better is the performance). Sanyal and Butler [24] presented a numerical study of power generation prospects from EGSs based on the multiple parallel fracture concept. The study used the heat recovery factor as a criterion for measuring the effectiveness of an EGS power project, and the results showed that the recovery factor was in the range of 0.4 to 0.5 . 
Among various numerical models of EGS fractured reservoir, the equivalent porous medium (EPM) model may be the most widely used one [25], in which the equivalent porosity/permeability of fractured reservoir can be determined from the distribution, orientation, and density of the fractures [26]. Specially, for a reservoir with parallel planar fractures, the equivalent permeability can be calculated by the cubic law of seepage flow [27]. Though the EPM model is competent for the simulation of heterogeneous reservoir, the reservoir is often assumed as a homogeneous porous medium in which the porosity and permeability in the reservoir volume are constant. This approach simplifies the specification of the parameter during mathematical modeling; the homogeneous porous model is widely used in the quantitative analysis of EGS processes. For some simple-structured EGS (e.g. 2-well structure and 5-well structure) with homogeneous reservoir, the heat extraction process can be predicted with given injection rate, injection temperature, reservoir temperature, and well separation distance [28]. Nakanishi et al. [29] compared the rate of cooling in a homogeneous medium and a fractured medium with $50 \mathrm{~m}$ or $100 \mathrm{~m}$ fracture spacing, the result indicated that the homogeneous medium gave a better heat extraction performance than the fractured medium; the results also implied that such difference might be neglected if the fracture spacing was small enough.

Since channeling and highly preferential flow paths commonly exist in actual reservoirs, the heat extraction performance of EGSs could be much lower than that predicted by models based on homogeneous porous reservoir or parallel fracture reservoir [30]. Garg and Combs [31] used the volumetric estimation method together 
with Monte Carlo simulations to estimate the heat recovery factors for naturally fractured geothermal systems and found the heat recovery factor was between $5 \%$ and $15 \%$, while that predicted by models with simplified reservoir assumptions was between $35 \%$ and $70 \%[24,32,33]$. The seepage flow pattern and residence time of heat transfer fluid in heterogeneous reservoir are different from those in homogeneous reservoir [34]. Therefore, it is necessary to evaluate the effects of reservoir heterogeneity on EGS heat extraction performance.

Researchers working on reservoir modeling have established many methods for reconstructing the reservoir of heterogeneous structure [35-40]. Kalinina [36] and Shaik et al. [37], based on the fracture orientation, spacing, aperture, and length, derived the distribution data of permeability and porosity in a heterogeneous reservoir. Llanos et al. [38] and Doonechaly et al. [41] converted the deterministic discrete fracture network in an EGS reservoir into the heterogeneous distribution data of permeability. Vogt et al. [35, 40] estimated the 3-D permeability field based on the tracer circulation data using Monte Carlo Method and Ensemble Kalman Filter method. However, the reliability and accuracy of the reconstructed reservoir, to a great extent, rely on the effectiveness and amount of the field test data such as the microseism measurement data, which are commonly obtained from a special EGS field site, consequently lacking universality.

\subsection{Objective of the present work}

The present work is aimed to obtain a deep understanding on the effects of 
reservoir heterogeneity on EGS heat extraction performance. We consider first a layered porous reservoir model, in which the permeable zone consists of a certain number of horizontal layers with each layer having a single porosity and permeability; in this way a parameter that can quantify the heterogeneity of reservoir is derived and defined. The heterogeneity of the layered porous reservoir is fully represented by the permeability array. To reduce the number of participating factors, we find a parameter that is able to quantify the EGS heat extraction performance and is solely dependent on the heterogeneity of reservoir, enabling to quantify the effects of reservoir heterogeneity. Based on the analysis on the simulation results from a large quantity of EGS cases, evaluation on the effects of reservoir heterogeneity is made. We then extend the results from idealized (layered) reservoir models to EGSs of more realistic heterogeneous reservoirs and discuss the applicability of the obtained relationship of EGS heat extraction performance versus reservoir heterogeneity.

\section{Methodology}

We have developed a transient, three-dimensional numerical model for the simulation of EGS long-term heat extraction processes $[42,43]$. The model treats the geothermal reservoir as a porous medium, while considers local thermal non-equilibrium between the solid rock matrix and fluid flowing in the fractures to simulate the local convective heat exchange in the porous reservoir. Recently, this model was used for several further studies. Chen and Jiang [34] presented a detailed 
study of the heat extraction process in EGSs with different reservoir permeability and volumetric heat exchange coefficient between rock and fluid. Chen and Jiang [32] analyzed the effects of well layout on multi-well EGS heat extraction performance and assessed the recoverable heat of EGS geothermal resource. Cao et al. [44] extended the model by implementing pressure- and temperature- dependent thermophysical properties of water and supercritical carbon dioxide. The numerical modeling and solution strategy in this study are the same as in previous works [29, 31 , 39-41], except that we assign location-dependent porosity $(\varepsilon)$ and permeability $(k)$ in the reservoir instead of using a homogeneous reservoir of constant porosity and permeability.

\subsection{Physical model}

The EGS subsurface geometry is treated as a single-domain consisting of multiple sub-regions [42, 43], namely: i) region 1 represents the heat reservoir; ii) region 2 the rock enclosing the heat reservoir; iii) region 3 the injection and production wells, as schematically displayed in Figure 1. The heat reservoir is taken as a porous medium with location-dependent porosity and permeability distribution. Different regions have distinct geo-physical properties. The rock enclosing the heat reservoir is impermeable to fluid, i.e. $\varepsilon=0$ and $k=0$, which means the probably occurring fluid loss during EGS heat extraction is not considered; the injection and production wells are treated as open channels, i.e. $\varepsilon=1$ and $k=\infty$. 


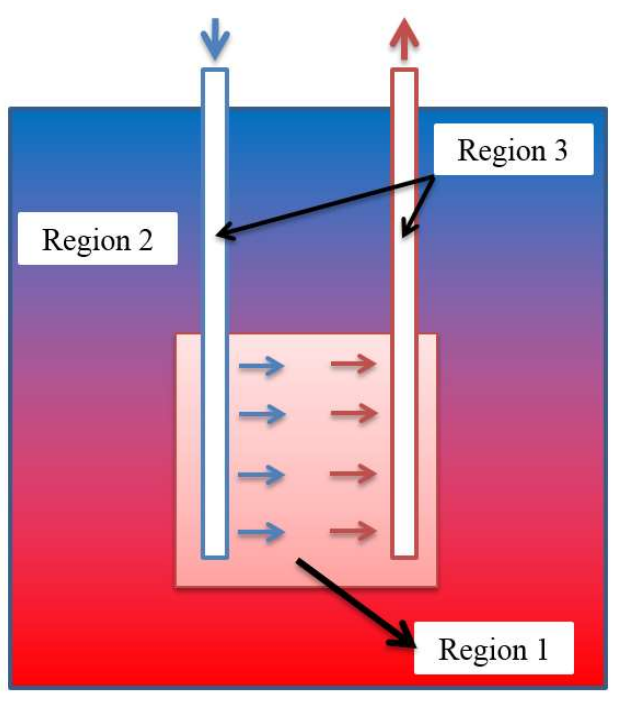

Figure 1 Physical model of EGS subsurface part.

\subsection{Numerical model}

The model is focused on modeling and analyses of the subsurface thermo-hydraulic process in EGS. The assumptions of the model have been detailed in previous works, say Refs. [42, 43]. The governing equations describing the conservation of mass, momentum and energy are formulated as the following.

Conservation of mass (mass continuity equation):

$$
\frac{\partial(\varepsilon \rho)}{\partial t}+\nabla(\rho \boldsymbol{u})=0
$$

Conservation of momentum:

$$
\frac{\partial(\rho \boldsymbol{u})}{\partial t}+\frac{\rho}{\varepsilon} \boldsymbol{u} \cdot \nabla \boldsymbol{u}=-\nabla P-\frac{\mu}{k} \boldsymbol{u}
$$

Energy equation in the fluid:

$$
\frac{\partial\left(\varepsilon \rho c_{p} T\right)_{\mathrm{f}}}{\partial t}+\nabla \cdot\left[\left(\rho c_{p} T\right)_{\mathrm{f}} \mathbf{u}\right]=\nabla \cdot\left(\lambda_{\mathrm{f}}^{e f f} \nabla T_{\mathrm{f}}\right)+h a\left(T_{\mathrm{s}}-T_{\mathrm{f}}\right)
$$


Energy equation in the rock or rock matrix in the porous reservoir:

$$
\frac{\partial\left[(1-\varepsilon) \rho c_{p} T\right]_{\mathrm{s}}}{\partial t}=\nabla \cdot\left(\lambda_{\mathrm{s}}^{e f f} \nabla T_{\mathrm{s}}\right)-h a\left(T_{\mathrm{s}}-T_{\mathrm{f}}\right)
$$

$\boldsymbol{u}, p, T_{\mathrm{f}}$ and $T_{\mathrm{s}}$, are the primary variables to be solved, denoting the superficial fluid velocity vector, fluid pressure, fluid temperature and rock temperature, respectively. $\rho$ and $c_{\mathrm{p}}$ with subscript of "s" and " $f$ " respectively signify the density and specific heat capacity of fluid and rock. $\mu$ is the viscosity of fluid. The heat conductivity of fluid and rock matrix in the reservoir are implemented with the effective quantity $\lambda^{\text {eff }}$, i.e.

$\lambda_{\mathrm{s}}{ }^{\mathrm{eff}}=\lambda_{\mathrm{s}}(1-\varepsilon)^{1.5}$ and $\lambda_{\mathrm{f}}{ }_{\mathrm{eff}}^{\mathrm{ef}}=\lambda_{\mathrm{f}} \varepsilon^{1.5}$, where $\varepsilon$ denotes the local porosity in the reservoir. Note that the Bruggemann approximation has been implemented here, which assumes effective transport property can be approximated by the intrinsic physical property of the medium multiplied by the component volume fraction being raised to power 1.5. The last terms of Eqs. (3) and (4), $\pm h a\left(T_{\mathrm{s}}-T_{\mathrm{f}}\right)$, describe the heat exchange between rock matrix and heat transfer fluid, where the parameter $h a$ denotes the volumetric heat exchange coefficient between rock matrix and fluid in the fracture.

\subsection{Model parameters}

The standard EGS doublet (one injection well and one production well) is considered for modeling configuration, as depicted in Figure 2 along with its dimensions. The computational domain is a $2000 \times 6000 \times 2000 \mathrm{~m}$ volume. The subsurface reservoir considered in this particular work is a $500 \times 500 \times 500 \mathrm{~m}$ cubic volume if not explicitly stated, and it is located at a depth of $4000 \mathrm{~m}$. The injection and production wells are all $0.2 \times 0.2 \mathrm{~m}$ square-shaped on the $x y$-sectional planes. The 
mesh used to discretize the whole computational domain employs 270,000 structural hexahedral grid elements. Grid refinements are made for the injection and production well regions of the reservoir to ensure sufficient resolution to accurately describe the physical processes. Grid-independence tests were conducted in previous works, say Ref. [32], which indicates that the present mesh system can give solutions of adequate accuracy.

The earth-surface temperature is fixed at $300 \mathrm{~K}$; the initial temperature of rock is considered as increasing linearly with the reservoir depth by $4 \mathrm{~K}$ for each $100 \mathrm{~m}$; the outer boundaries of region 2 are assumed adiabatic, which actually have no influence on the simulation results as the temperature evolution in the reservoir will not propagate to the boundaries of region 2 within the time-period (i.e. the lifetime of EGS) of interest. The circulating pump consumption is not taken into account in the performance evaluation of the present study; therefore, a constant mass flow rate is taken at the injection surface rather than a constant pressure condition. The injection rate is set at $75 \mathrm{~kg} / \mathrm{s}$ in most cases; however, it will be varied to be 37.5 or $150 \mathrm{~kg} / \mathrm{s}$ just for the purpose of conducting a sensitivity analysis concerning the injection rate.

In our previous works [29, 31, 39-41], the EGS reservoir is assumed to be homogeneously fractured with constant and uniform values of porosity and permeability; these values, based on the available literatures (refer to [32]), are 0.01 and $10^{-14} \mathrm{~m}^{2}$ for the porosity and permeability, respectively. In the present work, the 
porosity of each numerical volume element in the heat reservoir is assigned separately; while we restrict the average value of porosity to 0.01 to guarantee that the solid material in the reservoir, which is the same as saying the heat storage of the reservoir, remains unchanged for every single case. Meanwhile, the reservoir permeability is locally porosity-dependent, and calculated by the Verma-Pruess formula [45].

$$
\frac{k}{k_{0}}=\left(\frac{\varepsilon-\varepsilon_{c}}{\varepsilon_{0}-\varepsilon_{c}}\right)^{n}
$$

where, $k_{0}$ is taken as $10^{-14} \mathrm{~m}^{2} ; \varepsilon_{0}$ is taken as $0.01 ; \varepsilon_{c}$ is taken as $0.8 \varepsilon_{0} ; n$ is taken as 4 . The thermophysical properties of the fluid and rock are considered to be constant in this study.

Table 1 reports the thermophysical properties of the fluid and rock.
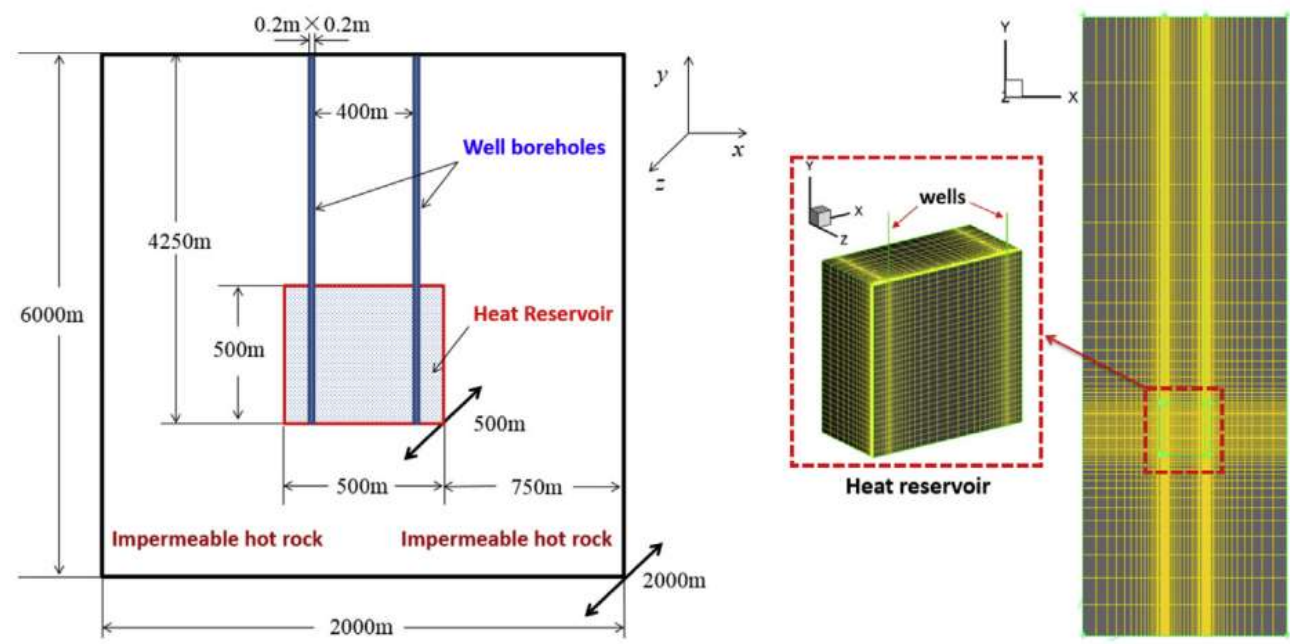

Figure 2 Geometrical dimensions and numerical mesh of the considered doublet EGS.

Table 1 Thermophysical properties 


\begin{tabular}{c|cccc}
\hline & $c_{p}(\mathrm{~J} / \mathrm{kg} / \mathrm{K})$ & $\lambda^{\text {eff }}(\mathrm{W} / \mathrm{m} / \mathrm{K})$ & $\rho\left(\mathrm{kg} / \mathrm{m}^{3}\right)$ & $\mu(\mathrm{Pa} \cdot \mathrm{s})$ \\
\hline Fluid & 4200 & 0.6 & 1000 & 0.001 \\
Rock & 1000 & 2.1 & 2650 & N/A \\
\hline
\end{tabular}

\section{Analysis of heat extraction from layered heterogeneous reservoirs}

To investigate the effects of heterogeneous reservoirs on EGS heat extraction performance, we consider three different reservoir permeability distributions with the permeable region located between $3850 \mathrm{~m}$ and $4350 \mathrm{~m}$ away from ground and they are: homogeneous reservoir of uniform permeability (HoR); layered heterogeneous reservoirs (HeR1 and HeR2), where HeR2 has an highly-preferential flow layer. The three distributions are depicted in Figure 3.

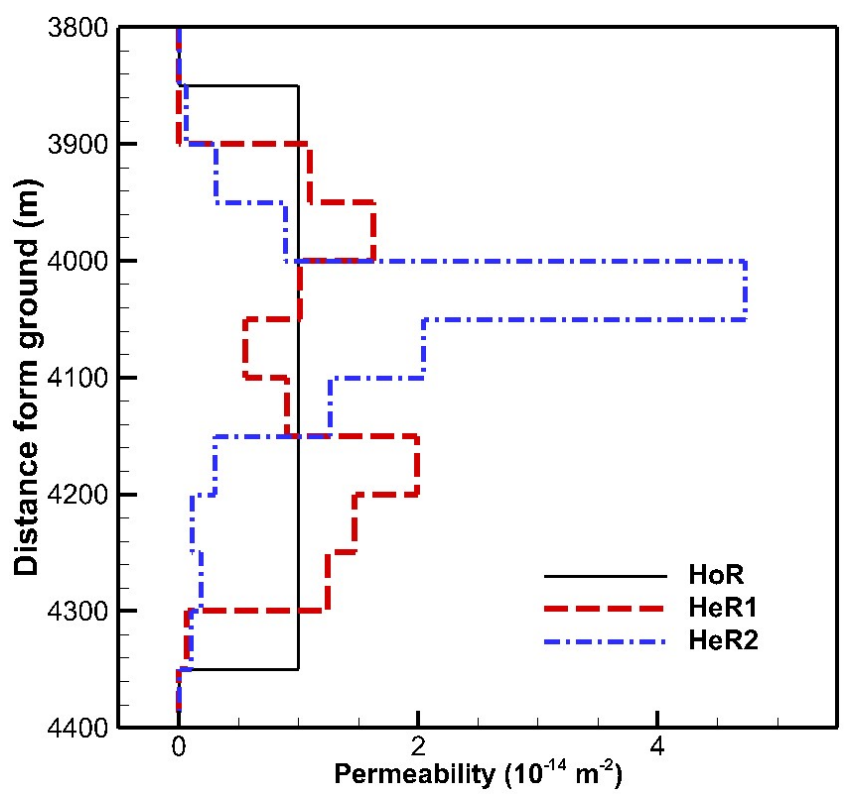

Figure 3 Profile of permeability against the reservoir depth direction for three typical categories of cases. 
The concept of "heat recovery factor", which is defined as the ratio of the extracted heat to the total heat stored in the reservoir volume, is commonly used as a criterion for the evaluation of EGS thermal performance $[24,32,33,46]$. In the present paper, thus, we define a similar factor, the heat extraction ratio $\gamma(\theta)$, as the criterion for the EGS thermal performance, which is defined as:

$$
\gamma(\theta)=\frac{\int_{0}^{t(\theta)}\left(T_{\text {out }}-T_{\text {in }}\right) \cdot\left(\rho c_{\mathrm{p}}\right)_{\mathrm{f}} \cdot Q_{\mathrm{v}} \cdot d t}{\int_{V}\left(T_{\mathrm{s}}-T_{\text {in }}\right) \cdot\left(\rho c_{\mathrm{p}}\right)_{\mathrm{s}} \cdot d V}
$$

where, $V$ is the volume of the reservoir and $Q_{\mathrm{v}}$ is the volumetric flow rate of the heat transmission fluid. The dimensionless temperature $\theta$ is used to express the production temperature decline, and it is defined as,

$$
\theta=\frac{T_{\text {out,max }}-T_{\text {out }}}{T_{\text {out,max }}-T_{\text {in }}}
$$

where, $T_{\text {out }}$ is the fluid production temperature; $T_{\text {in }}$ is the temperature of the injection fluid; $T_{\text {out,max }}$ is the maximum value of $T_{\text {out. }}$

We define the local heat extraction ratio $\gamma_{\mathrm{L}}(t)$ as:

$$
\gamma_{\mathrm{L}}(t)=\frac{T_{\mathrm{s}}-T_{\mathrm{s}}(t)}{T_{\mathrm{s}}-T_{\mathrm{in}}}
$$

While heat extraction progresses, the effectiveness of the EGS power generation will be declining as result of the diminishing production temperature level [9]. The 
EGS reservoir would have to be abandoned if the production temperature drops below a certain level, which is not suitable for power generation, meeting in this way the end of its service life. Different criteria of abandonment temperature are used in the literature $[24,46]$; they range from $5 \%$ to $15 \%$ decline of the production temperature compared with its maximum value. The heat extraction ratio, as defined by Eq. (6), gives the total extracted heat divided by the total heat stored in the heat reservoir when the production temperature drops to a particular level, and it is flexible for the evaluation under different criteria of abandonment temperature.

All the cases simulated in the present work are tabulated in Table 2.

Table 2 simulated cases

\begin{tabular}{|c|c|c|c|c|c|}
\hline Group \# & Case \# & $\begin{array}{l}\text { Reservoir } \\
\text { structure }\end{array}$ & permeability setting & $\begin{array}{c}\text { Mass } \\
\text { flow rate } \\
(\mathrm{kg} / \mathrm{s})\end{array}$ & $\begin{array}{c}\text { Reservoir } \\
\text { dimensions } \\
\left(l_{x} \times l_{y} \times l_{z}, \mathrm{~m}\right)\end{array}$ \\
\hline \multirow{3}{*}{1} & 1 & 10 layers & HoR & 75 & $500 \times 500 \times 500$ \\
\hline & 2 & 10 layers & HeR1 & 75 & $500 \times 500 \times 500$ \\
\hline & 3 & 10 layers & HeR2 & 75 & $500 \times 500 \times 500$ \\
\hline \multirow{6}{*}{2} & 4 & 10 layers & HoR & 150 & $500 \times 500 \times 500$ \\
\hline & 5 & 10 layers & HoR & 37.5 & $500 \times 500 \times 500$ \\
\hline & 6 & 10 layers & HeR1 & 150 & $500 \times 500 \times 500$ \\
\hline & 7 & 10 layers & HeR1 & 37.5 & $500 \times 500 \times 500$ \\
\hline & 8 & 10 layers & HeR2 & 150 & $500 \times 500 \times 500$ \\
\hline & 9 & 10 layers & HeR2 & 37.5 & $500 \times 500 \times 500$ \\
\hline \multirow{8}{*}{3} & 10 & 10 layers & Similar to HoR & 75 & $250 \times 250 \times 250$ \\
\hline & 11 & 10 layers & Similar to HoR & 75 & $250 \times 500 \times 500$ \\
\hline & 12 & 10 layers & Similar to HoR & 75 & $500 \times 250 \times 500$ \\
\hline & 13 & 10 layers & Similar to HoR & 75 & $500 \times 500 \times 250$ \\
\hline & 14 & 10 layers & Similar to HeR1 & 75 & $250 \times 250 \times 250$ \\
\hline & 15 & 10 layers & Similar to HeR1 & 75 & $250 \times 500 \times 500$ \\
\hline & 16 & 10 layers & Similar to HeR1 & 75 & $500 \times 250 \times 500$ \\
\hline & 17 & 10 layers & Similar to HeR1 & 75 & $500 \times 500 \times 250$ \\
\hline
\end{tabular}




\begin{tabular}{|c|c|c|c|c|c|}
\hline 4 & $18 \sim 47$ & 10 layers & $\begin{array}{l}\text { stochastic reservoir; } \\
\text { from } 1 \mathrm{e}^{-13} \text { to } 1 \mathrm{e}^{-15} \mathrm{~m}^{2}\end{array}$ & 75 & $500 \times 500 \times 500$ \\
\hline \multirow{3}{*}{5} & $48-56$ & 10 layers & \multirow{3}{*}{$\begin{array}{l}\text { stochastic reservoir; } \\
\text { from } 1 \mathrm{e}^{-13} \text { to } 1 \mathrm{e}^{-15} \mathrm{~m}^{2} \\
\text { stochastic reservoir; } \\
\text { from } 1 \mathrm{e}^{-13} \text { to } 1 \mathrm{e}^{-15} \mathrm{~m}^{2} \\
\text { stochastic reservoir; } \\
\text { from } 1 \mathrm{e}^{-13} \text { to } 1 \mathrm{e}^{-15} \mathrm{~m}^{2}\end{array}$} & 75 & $500 \times 500 \times 500$ \\
\hline & $57-65$ & 20 layers & & 75 & $500 \times 500 \times 500$ \\
\hline & $66-74$ & 40 layers & & 75 & $500 \times 500 \times 500$ \\
\hline 6 & $75-94$ & $\begin{array}{c}3 \mathrm{D} \\
\text { heterogeneous }\end{array}$ & $\begin{array}{l}\text { stochastic reservoir; } \\
\text { from } 1 \mathrm{e}^{-12} \text { to } 1 \mathrm{e}^{-16} \mathrm{~m}^{2}\end{array}$ & 75 & $500 \times 500 \times 500$ \\
\hline 7 & $95-114$ & $\begin{array}{c}3 \mathrm{D} \\
\text { heterogeneous }\end{array}$ & $\begin{array}{c}\text { same as group } 6 \text { cases } \\
\text { except } k_{\mathrm{y}}=0\end{array}$ & 75 & $500 \times 500 \times 500$ \\
\hline
\end{tabular}

\subsection{EGS heat extraction performance}

Three cases (i.e. case 1-case 3) were considered; the corresponding porosity distributions were presented in Figure 3. Case 1, Case 2 and Case 3 employ the reservoirs of HoR, HeR1 and HeR2, respectively. The difference in heat extraction performance of the three cases is reported in Figure 4, in which the horizontal coordinate is the operating time and the vertical coordinate contains two different operation indicators, namely: (1) production temperature; and (2) heat extraction ratio. 


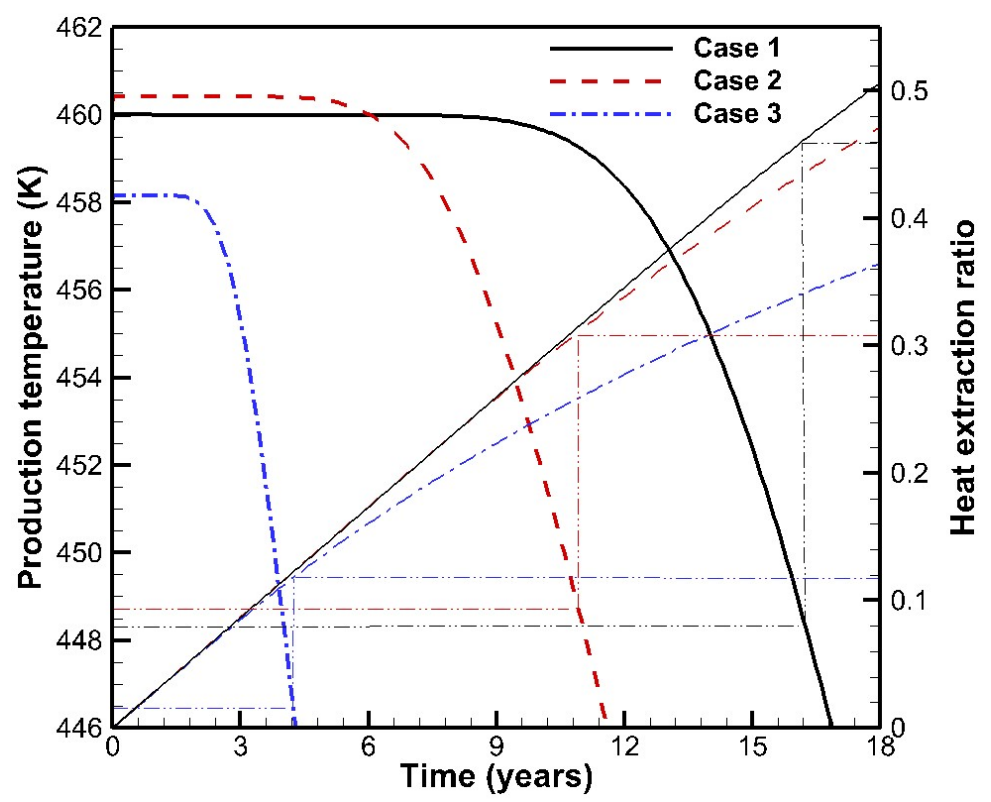

Figure 4 Effect of the reservoir heterogeneity on EGS production temperature and heat extraction ratio.

The production temperature as a function of the operating time indicates a significant difference in the heat extraction process for the three cases. In general, the production temperature remains at high level during the early years $(\sim 10$ years for HoR) of the heat extraction process and then decreases sharply. However, the time when temperature starts to decrease is rather different for the three cases; also the decreasing rate for Case 3 differs widely from those of the other two cases. If we take the EGS service life (or lifetime) when the dimensionless production temperature is $10 \%$ lower than the highest value (i.e., $\theta=0.1$ ), which is approximately the average of the values (from 0.05 to 0.15 ) encountered in the literature $[24,46]$, then, the service life for the three cases (Case 1-Case 3) are approximately 15.76, 10.68 and 4.04 years, respectively. By combining the lifetime indicator with the heat extraction ratio as a 
function of the operating time, we can find marked differences in performance for the three cases, which can be quantified by using the heat extraction ratio at the end of EGS service life. Its values for Cases 1, 2 and 3 are 0.45, 0.30 and 0.11, respectively. This result indicates that there are large differences in EGS heat extraction performance at the end of service life of the three considered cases, which is apparently correlated with the reservoir inner structures.

\subsection{Seepage flow and heat extraction process}

In Section 3.2, we have shown that there are large differences in EGS heat extraction performance at the end of service life for the three considered cases. In order to identify the cause for such behavior, we conducted a study on the seepage flow and heat extraction process for the three cases.

The seepage flow of the half-cut reservoir (the half-cut section lays in the middle of the reservoir and cross the two wells) in Cases 1, 2 and 3 is presented in Figure 5. In the figure, the surfaces of reservoir are colored depending on the magnitude of the velocity vector. Obviously, the horizontal flow (parallel to the $\mathrm{x}-\mathrm{z}$ plane and perpendicular to the flow in the wells) is dominant; while the vertical flow (parallel to the wells) is practically negligible. This observation indicates that, in the layered porous reservoir, there is negligible mass transfer between two adjacent layers. In a previous study [34], we have shown that there is little or no pressure change in the injection/production wells if the reservoir permeability is sufficiently low (i.e. $<10^{-12}$ $\mathrm{m}^{2}$ ) compared with the Darcy resistance in the reservoir. Taking into consideration 
that in these particular cases the permeability ranges from $10^{-13}$ to $10^{-15} \mathrm{~m}^{2}$, it makes sense to assume that the pressure in the wells remains constant along the depth of the reservoir for the three cases. Thus, based on the features of the seepage flow in a single layer, we can conclude that the seepage flow in a particular layer is only affected by the permeability of that layer.

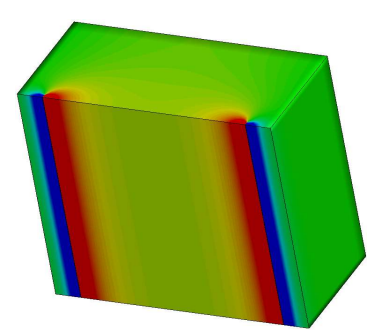

Case. 1
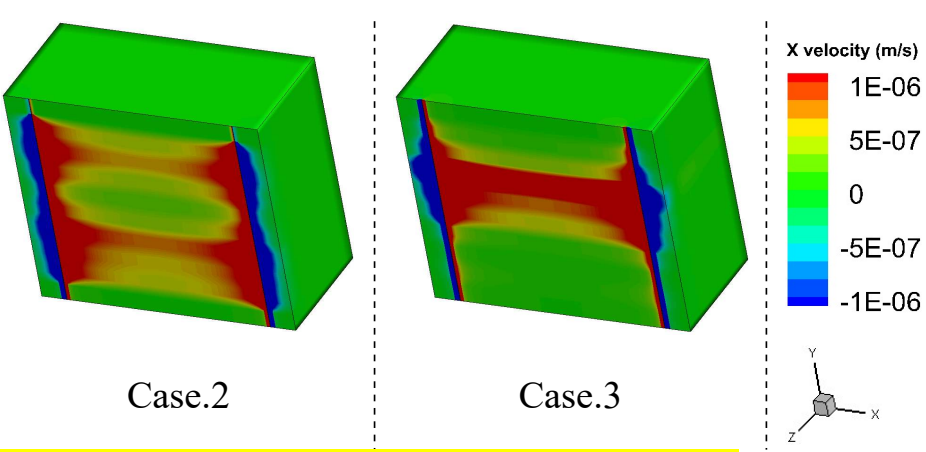

Figure 5 Seepage flow field for Cases 1, 2 and 3.

In order to show the relationship between the seepage flow and the heat extraction process in the reservoir, in Figure 6 is presented the distribution of temperature and local heat extraction ratio. Here we define the concept of "cold front" as the surface made by all the reservoir numerical elements in which the local heat extraction ratio is equal to 0.01 . We also define the "thermal breakthrough" as the time point when the "cold front" reaches any part of the production well, which marks the beginning of the production temperature drop. All diagrams presented in Figure 6 are taken at the time for which "thermal breakthrough" just occurs, namely, 10.49, 6.18, 2.28 years for Cases 1, 2 and 3, respectively. From close observation of Figure 5 and Figure 6, it can 
be easily noticed that there is a strong relation between the velocity of seepage flow and the forward movement of the "cold front". In Case 1, since the seepage flows in all the layers share the same velocity, the movement of "cold front" shows little difference along the well-depth direction and it sweeps a large volume of reservoir. In contrast, in Case 2 and Case 3 where the uneven seepage flow exists along the well-depth direction, the "cold front" in the preferential flow layer reaches the production well at an earlier time, causing an earlier "thermal breakthrough" and leading to a smaller amount of heat extraction. The preferential flow for case 3 is more severe than case 2 , the "thermal breakthrough" in case 3 is thus seen to take place at a much earlier time point.

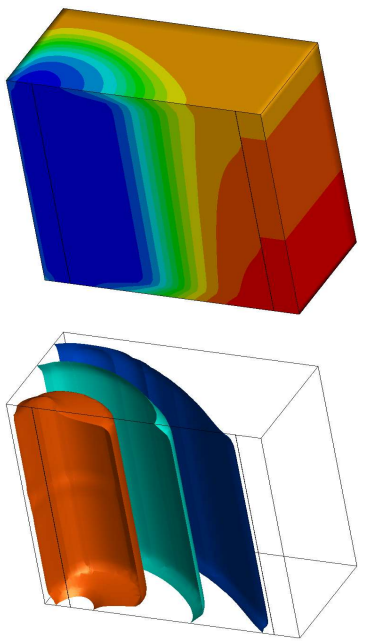

Case 1@10.49 years
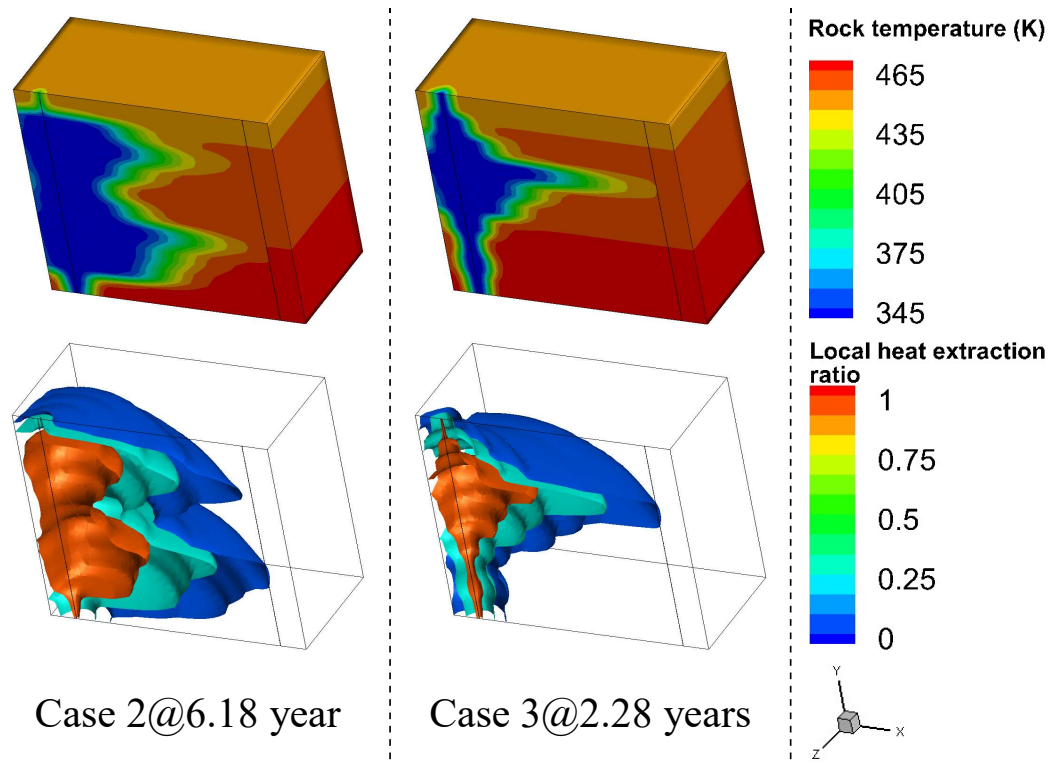

Figure 6 Rock temperature and local heat extraction ratio for Cases 1,2 and 3 at thermal breakthrough. 
In addition, we attempt to present the quantified heat extraction performance, seepage flow and reservoir heterogeneity in Figure 7Figure 3. The fluid shortest residence time is presented in the horizontal coordinate, and it has an inverse-ratio correlation with the maximum flow flux in the layered reservoir. It is a feature of the seepage flow, and it reflects the short-circuit level in the seepage flow field. Two vertical coordinates are used in this figure: (1) EGS heat extraction ratio (when $\theta=$ 0.01 or $\theta=0.1$ ); and (2) $k_{\text {ave }} / k_{\max }$, where, $k_{\text {ave }}$ is the volumetrically-averaged permeability in the reservoir which is defined by Eq. (9), namely:

$$
k_{\text {ave }}=\frac{\int_{0}^{l_{y}} k d y}{l_{y}}
$$

and $k_{\max }$ is the maximum permeability of all the layers in the reservoir. In Eq. (9), $l_{\mathrm{y}}$ is the height of the reservoir. Consequently, $k_{\text {ave }} / k_{\max }$ to some extent reflects the reservoir heterogeneity. 


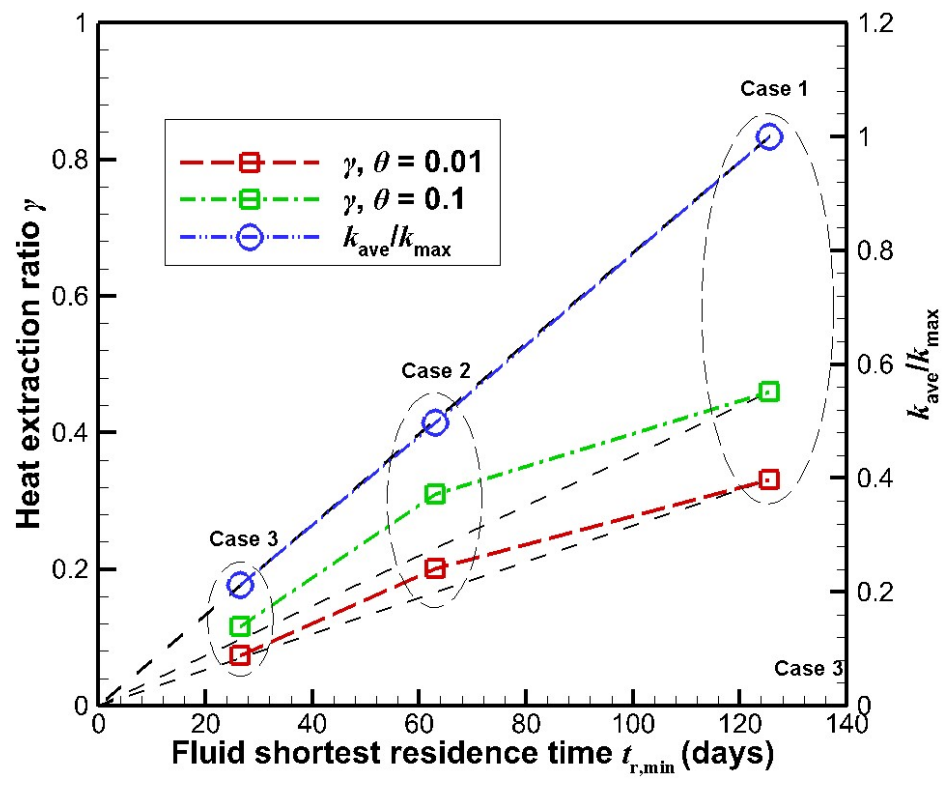

Figure 7 Heat extraction ratio and the short-circuit factor $\left(k_{\mathrm{ave}} / k_{\max }\right)$ versus the fluid shortest residence time for Cases 1, 2, and 3.

As shown in Figure 7, for cases 1 and 3, the shortest residence time versus the EGS heat extraction ratio have very similar trends for different heat-mining stages. This indicates the heat extraction process for heterogeneous and homogeneous EGS cases, most likely, can be scaled by the characteristics (e.g. the fluid shortest residence time) of the fluid seepage flow. However, Case 2 shows clear deviation from this phenomenon and this deviation increases with increasing values of $\theta$. This indicates the fluid shortest residence time is not universally competent for representing the heterogeneity of EGS reservoirs.

In Figure 7 it can be also noted that the ratio of $k_{\text {ave }} / k_{\max }$ versus the fluid shortest residence time falls on a straight line for all the threes cases, which is in agreement with previous results, as we have discussed when analyzing Figure 5, that the seepage 
flow in a single layer is only affected by the permeability of this layer. That is to say, the features of the seepage flow can be used to characterize the reservoir heterogeneity.

\section{Quantifying the effects of reservoir heterogeneity}

From the previous section 3, it was found that the reservoir heterogeneity can lead to a marked decline of EGS heat extraction performance. In this section, we will quantify the relation between EGS heat extraction performance and the reservoir heterogeneity.

\subsection{Relative heat extraction ratio $(\eta)$}

In order to quantify the effects of reservoir heterogeneity, it is required to eliminate the influence of other intervening factors. The heat extraction ratio $\gamma(\theta)$ gives the reservoir heat utilization status, and it can be used to evaluate the heat extraction performance of the EGSs. However, the simulation results (obtained from cases 1-17) shown in Figure 8 indicate that $\gamma(\theta)$ is clearly affected by the injection flux and reservoir dimensions. It is obvious that the heat extraction ratio should not be directly used as the criterion for the evaluation of the reservoir heterogeneity effects. 


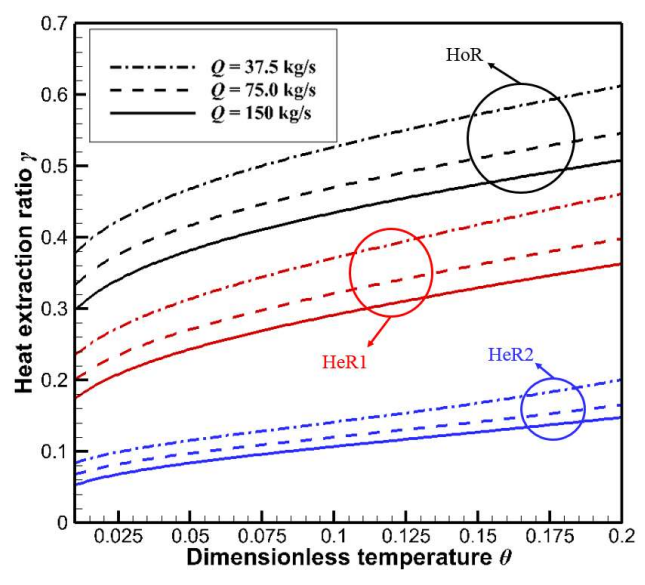

(a)

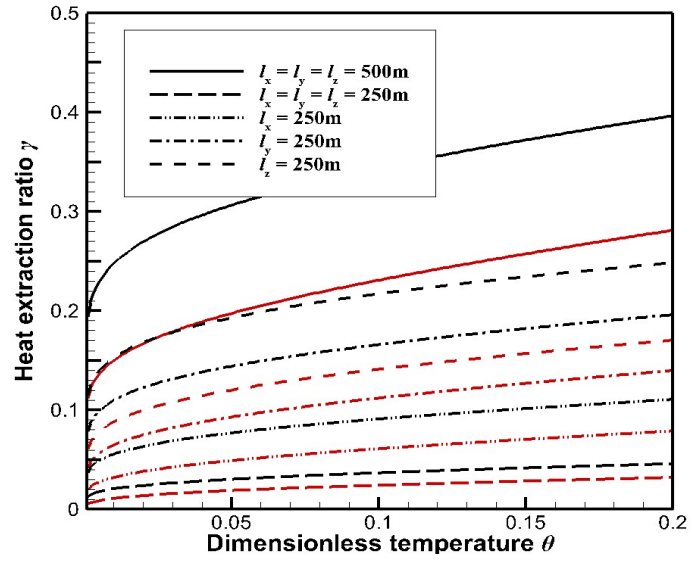

(b)

Figure 8 Effects of injection flux (a) and reservoir dimensions (b) on heat extraction ratio.

Therefore, a new parameter, the relative heat extraction ratio $\eta$, is defined as:

$$
\eta(\theta)=\frac{\gamma(\theta)}{\gamma_{0}(\theta)}
$$

where, $\gamma_{0}(\theta)$ is the heat extraction ratio from the HoR case when the production temperature is $\theta$.

As shown in Figure 9, the relative heat extraction ratio shows little dependence on the injection flow rate and the reservoir dimensions, and can be approximately looked as a parameter solely depending on the reservoir heterogeneity. It is more suitable to use the relative heat extraction ratio $\eta$ to evaluate the change in EGS heat extraction performance caused by reservoir heterogeneity. The relation between $\eta$ and the reservoir heterogeneity can be deduced from simulation results of a large quantity of cases with different reservoir heterogeneity values, and it is possible to predict the 
$\eta$ of an EGS of a certain heterogeneous reservoir according to its reservoir heterogeneity. Once the value of $\eta$ is determined, the EGS heat extraction performance can be estimated based on the heat extraction ratio for HoR EGS. Hence, a quantified evaluation on the effects of reservoir heterogeneity could be made.

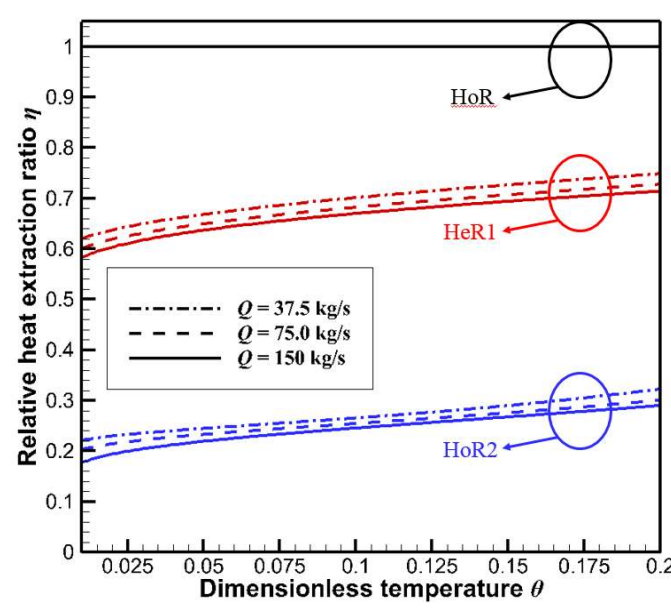

(a)

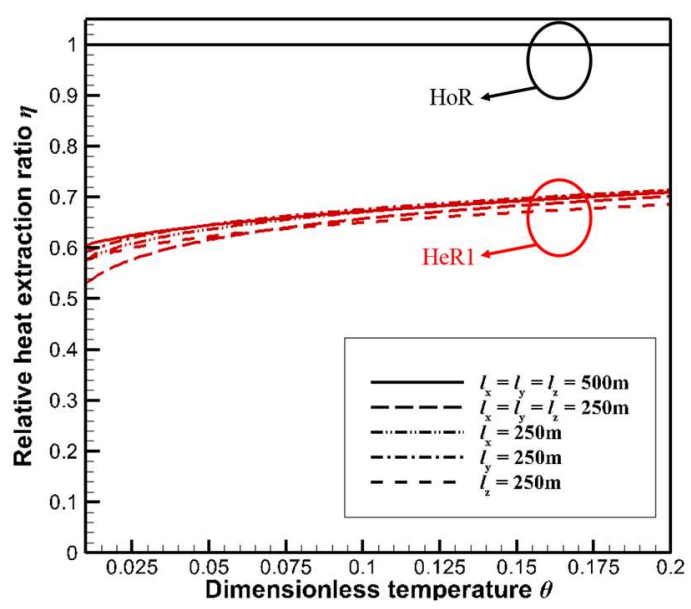

(b)

Figure 9 Effects of injection flux (a) and reservoir dimensions (b) on the relative heat extraction ratio.

\subsection{Reservoir heterogeneity $(\alpha)$}

As already mentioned in the analysis of Figure 7, the shortest residence time of fluid and the EGS heat extraction ratio have a non-linear relation: the ratios of the fluid shortest residence time to the EGS heat extraction ratio are the same between the cases concerning the homogeneous reservoir (HoR) and the highly heterogeneous reservoir (HeR2); however, the slightly heterogeneous reservoir (HeR1) deviates from 
this relation, moreover, the deviation increases with an increasing value of $\theta$.

It is not difficult to understand these observations as: 1) in the highly heterogeneous reservoir (HeR2), the seepage flow peaks in the region with the highest permeability and, consequently, it forms an apparent preferential flow; 2) the flow passing through the preferential flow region makes up for the most of the production well flow, thus the performance of system is dominated by the features of preferential flow (e.g. the shortest residence time of fluid); and 3) in the slightly heterogeneous reservoir (HeR1), the flow going through the preferential flow region is relatively low, consequently, the performance of the system is likely determined by the averaged features of fluid flow in the whole reservoir. In order to better comprehend this phenomenon, a sufficiently large number of cases are required for the analysis. For this reason, we built and simulated 30 stochastic layered heterogeneous reservoir cases (cases 18-47 listed in Table 2) and define two dimensionless factors, namely:

$$
\begin{gathered}
\alpha_{1}=\frac{k_{\text {ave }}}{k_{\max }} \\
\alpha_{2}=\frac{k_{\text {ave }}}{k_{\text {mass,ave }}}
\end{gathered}
$$

$k_{\text {mass,ave }}$ is an averaged reservoir permeability with the mass flow rate of fluid $(Q)$ as the weight factor, namely:

$$
k_{\text {mass,ave }}=\frac{\int_{Q} k d Q}{Q}
$$


In Figure $7, \alpha_{1}$ (defined as $k_{\text {ave }} / k_{\max }$ ) looks to be directly proportional to the shortest residence time of fluid; therefore, it is reasonable to expect that $\alpha_{2}$ should well represent the averaged residence time of fluid. Based on the simulation results from the 30 cases (i.e. cases 18-47), these two factors $\left(\alpha_{1}\right.$ and $\left.\alpha_{2}\right)$ versus the relative heat extraction rate $\eta$ are reported in Figure 10.

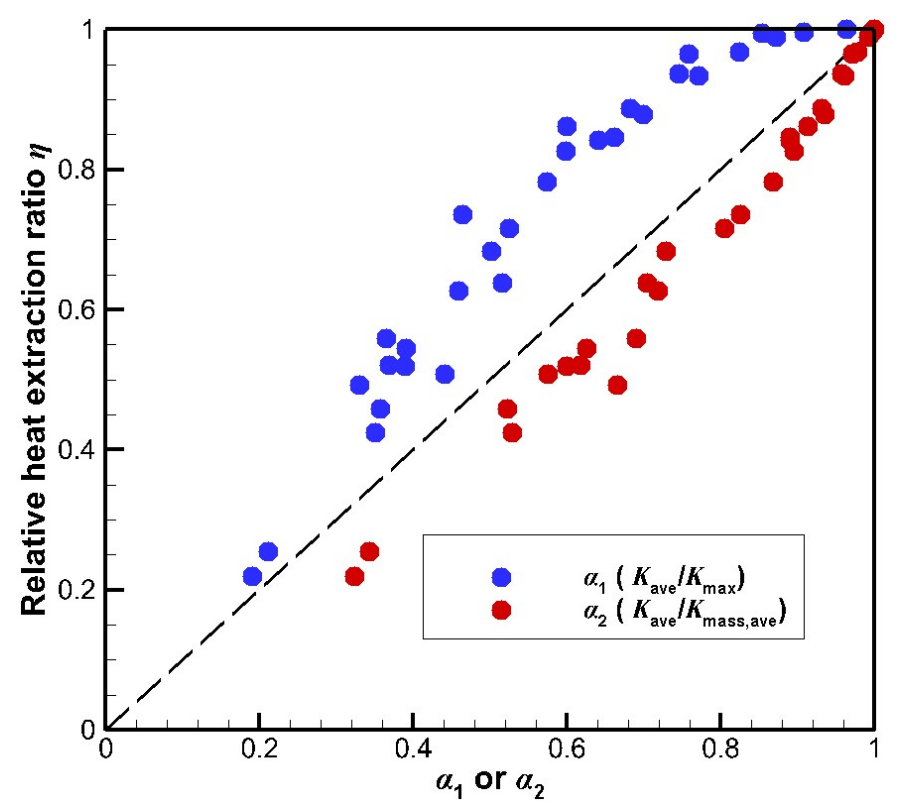

Figure 10 The two dimensionless factors $\left(\alpha_{1}\right.$ and $\left.\alpha_{2}\right)$ versus the relative heat extraction ratio $\eta$.

In Figure 10, the dashed diagonal line is taken as a standard line, which means that the factors are equal to the relative heat extraction ratios. As it is shown in Figure $10, \alpha_{1}$, which represents the shortest residence time of fluid, tends to be lower than the relative heat extraction ratio. However, $\alpha_{2}$, which represents the 
mass-flow-rate-averaged residence time of fluid, tends to be higher than the relative heat extraction ratio. These observations indicate that the overall residence time of fluid in a heterogeneous reservoir is longer than that in the preferential flow region and shorter than that calculated in terms of the fluid average velocity.

Under these conditions, we propose to define the reservoir heterogeneity using a combination of $\alpha_{1}$ and $\alpha_{2}$, namely:

$$
\alpha=\frac{1}{\sqrt[n]{\frac{\int\left(\frac{k}{k_{\text {ave }}}\right)^{n} d Q}{Q}}}
$$

where, $n$ represents an adjustment index $(n \geq 1)$, the value of which can adjust the sensitivity of $\alpha$ to the preferential flow in the reservoir, as:

$$
\alpha=\left\{\begin{array}{l}
\alpha_{1}, n \rightarrow \infty \\
\alpha_{2}, n=1
\end{array}\right.
$$

Note that the reservoir heterogeneity, defined by Eq. (14), monotonically decreases with the increase of $n$. According to Eq. (15), we know that $\alpha$ takes values within a range of $\left(\alpha_{1}, \alpha_{2}\right)$, when $n \in(1, \infty)$.

Figure 11 presents the relation between the reservoir heterogeneity and the relative heat extraction ratio for different values of the adjustment index $n$. It can be deduced that by using an appropriate value for the adjustment index $n$, there is a very simple relation between $\eta$ and $\alpha$, namely:

$$
\eta=\alpha
$$




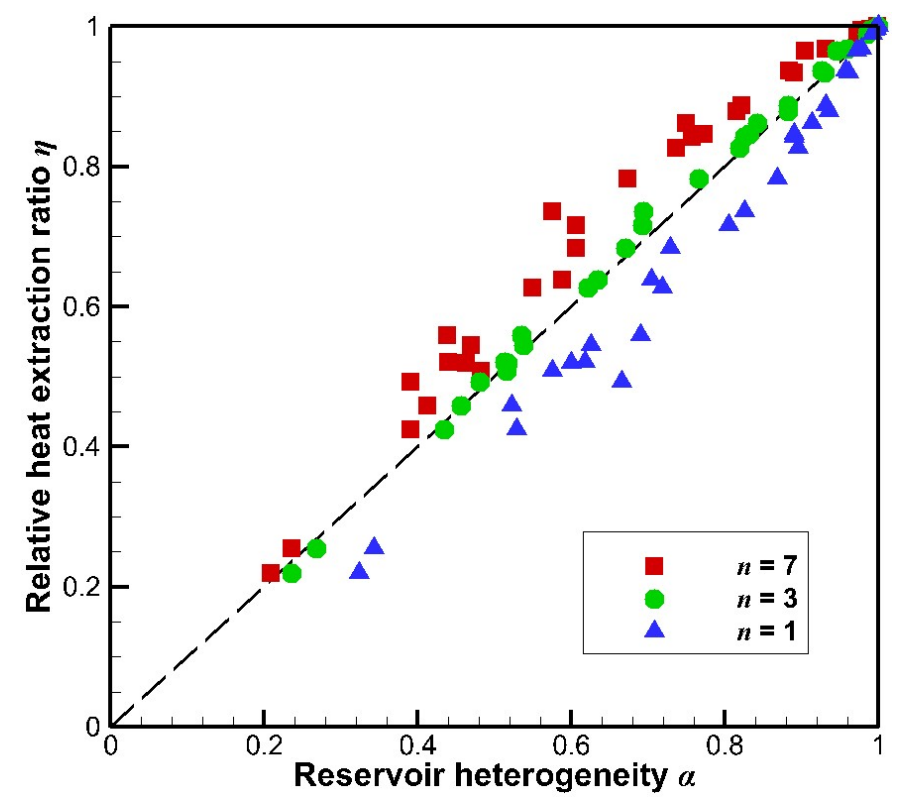

Figure 11 Relation between the reservoir heterogeneity and the relative heat extraction ratio for different values of the adjustment index $n$.

\subsection{Adjustment index (n)}

For a given case, the relative heat extraction ratio $\eta$ will change with the change of dimensionless temperature $\theta$. When calculating the reservoir heterogeneity $\alpha$, the adjustment index $n$ should be changed in accordance with the dimensionless temperature $\theta$ to obtain the best fit using Eq. (16). Thus, the formulation of the relation between dimensionless temperature $\theta$ and optimum adjustment index $n_{\theta}$ is a must-be-solved problem. We investigate this problem through the simulation results of the 30 SLHeR (Stochastic Layered Heterogeneous Reservoirs) cases, i.e. cases 18-47 listed in Table 2. 
The results are organized into Figure 12 in which a colored contour map of R-square is used to show the fitting degree of the simulation results in relation to Eq. (16).

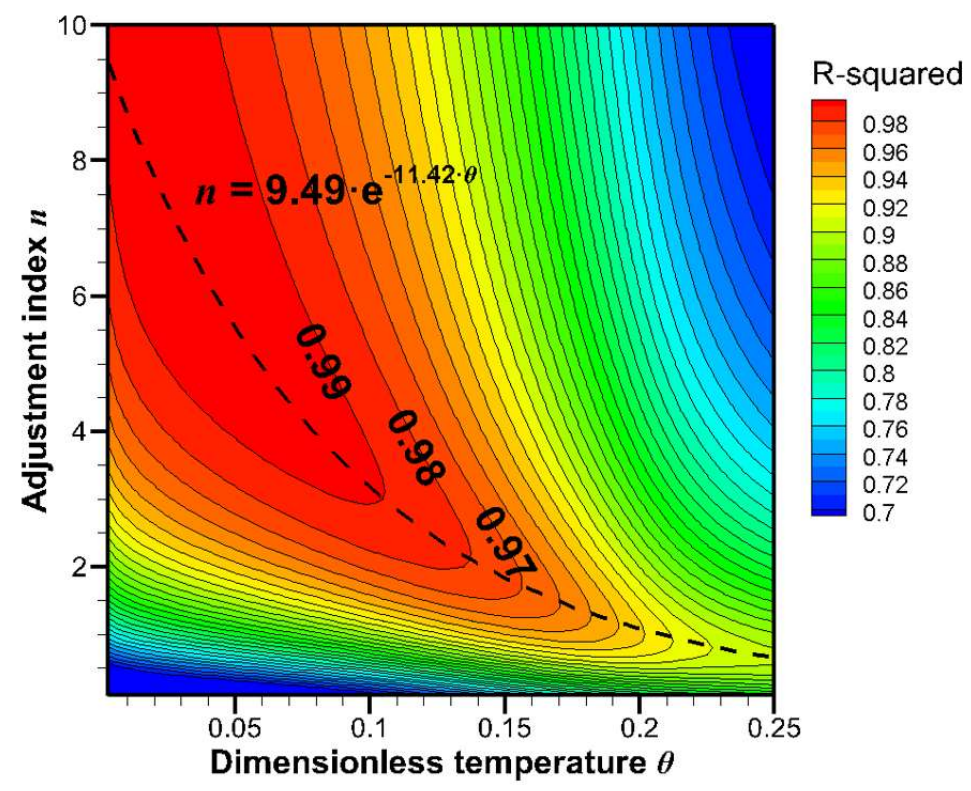

Figure 12 R-square values for the fitting Eq. (16) for different dimensionless temperature values and adjustment index $n$.

Figure 12 indicates that with increasing values of $\theta$, decreasing values of $n$ should be used in order to get the best fit; when the dimensionless temperature $\theta$ is over 0.15 , it is not viable have a very well fit (R-square greater than 0.97$)$. In general, the relation between the dimensionless temperature $\theta$ and the optimum adjustment index $n_{\theta}$ can be described by the following expression:

$$
n_{\theta}=9.49 e^{-11.42 \theta}
$$


Based on this relation, the value of $\theta$ reflects the extent of heat extraction process and the value of adjustment index $n$ reflects the sensitivity of reservoir heterogeneity $\alpha$ to the seepage velocity of preferential fluid flow. Therefore, the relationship between $\theta$ and $n$ reported in Figure 12 indicates that along with the heat-mining, the EGS heat extraction performance becomes less sensitivity to the preferential flow in reservoir. Note that the curve of $n_{\theta}$ versus $\theta$ is depicted as a dashed line in Figure 12.

As the heat-mining is progressing, thermal breakthrough will first occur in the preferential flow path, and the temperature of fluid passing through preferential flow path will drop, which will lead to a temperature decline in the production well. In the early stages of the heat-mining process prior to the occurrence of thermal breakthrough, the preferential flow has a significant influence on the reservoir heat extraction performance. As a result, a higher value of the adjustment index $n$ should be taken for achieving a good match between the simulation data and the predictions by Eq. (16). When the heat-mining process comes to an advanced stage after the occurrence of thermal breakthrough, the influence of the preferential flow will become relatively weak, in this way requiring a smaller adjustment index $n$ to get a good match between simulations and the predictions by Eq. (16).

In the final stages, the effect of heat conduction between high permeability region and low permeability region tends to become increasingly relevant. Therefore, assuming that the heat extraction ratio is similar to the seepage flow rate is most likely not appropriate, and it will lead to a mismatch between the simulation data and the 
predictions by Eq. (16).

\subsection{Effect of layer thickness}

All the above-described simulated cases considered EGS reservoirs of 10 layers. One additional series of 27 stochastic layered heterogeneous reservoir cases (i.e. cases 48-74 listed in Table 2) were built. In these cases, the reservoir is divided into several equal layers; the porosity of each layer is assigned randomly using a random number generator and its value varies in the range of 0.008 to 0.012 while the mean value of all the layers is kept at 0.01 ; the permeability calculated by Eq. (5) ranges from $10^{-13}$ to $10^{-15} \mathrm{~m}^{2}$. According to the number of layers in the reservoir, the cases are divided into three sub-groups (having 10, 20, and 40 layers, respectively), and each sub-group contains 9 cases. As all the reservoirs have the same height in the well-depth direction, different layer number means different layer thickness considered.

The calculated results are shown in Figure 13. In this figure, the data points for the different cases and prediction lines by Eq. (16) are displayed for different dimensionless temperature. Overall, the method shows good predictability. The predictions can fit well the simulation results for small dimensionless production temperature. However, the prediction becomes increasingly inaccurate when the dimensionless temperature increases. As already discussed, thermal conduction between layers in the heterogeneous reservoir will improve the heat extraction performance, and its effect tends to become more and more significant with the progress of heat extraction. Meanwhile, because of thermal conduction between 
layers, the 40-layer sub-group having the thinnest layer tends to reach a higher value of $\eta$ than the other two sub-groups, and this trend becomes increasingly apparent as the dimensionless temperature increases.
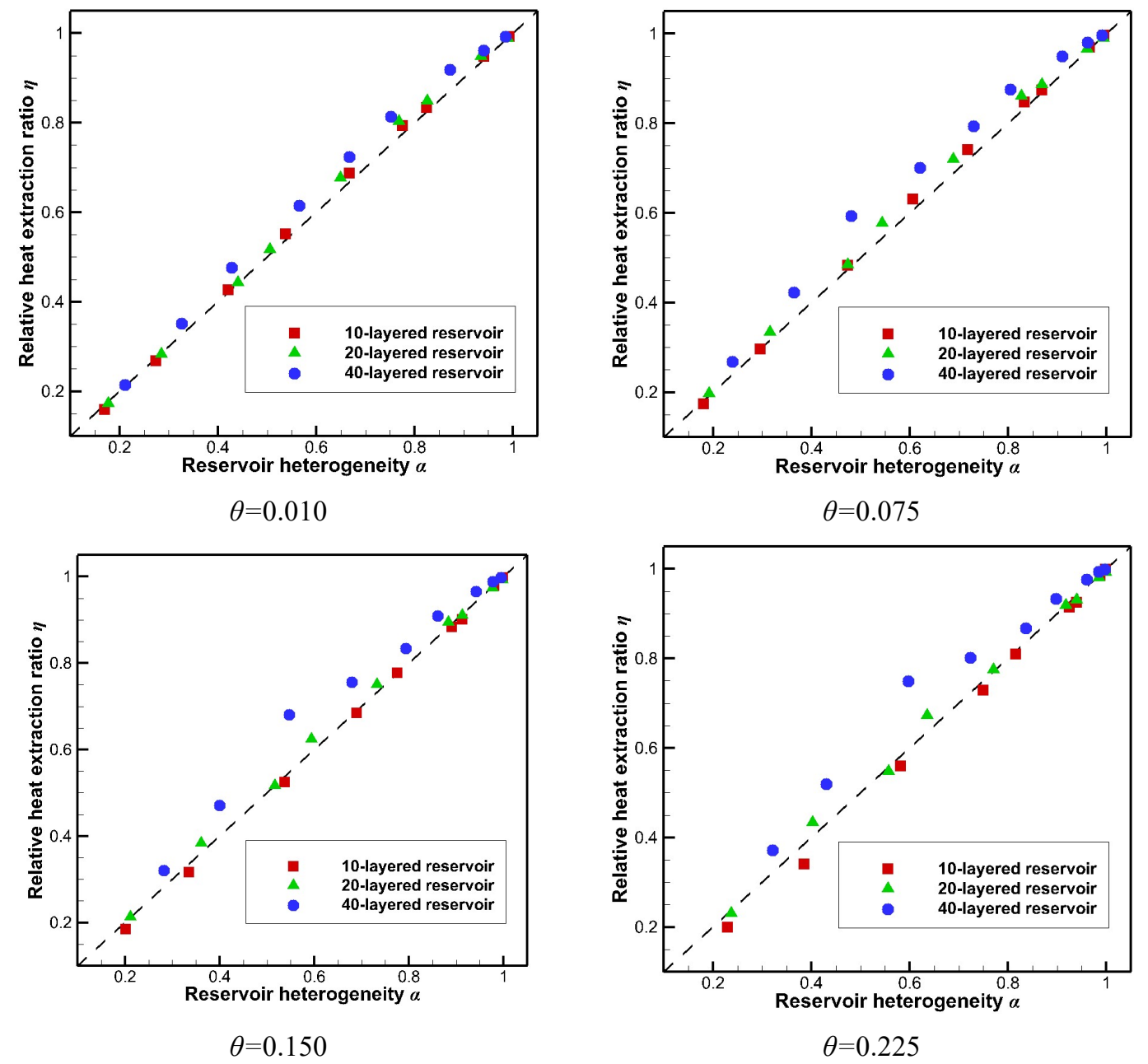

Figure 13 Predicted values by Eq. (16) compared to simulation results for cases 48-74 at different dimensionless production temperature.

\section{Application to more practical heterogeneous reservoirs}

The reservoir heterogeneity defined by Eq. (14) is based on the permeability 
distribution in EGS heat reservoir. For practical use, since there is almost no possibility to determine the permeability distribution inside a real EGS reservoir, we propose an equivalent approach to calculate the reservoir heterogeneity. In this equivalent approach, the fluid mass-flow rate profile (which is measurable) along the injection well-depth direction is employed to calculate the reservoir heterogeneity.

Fluid mass-flow rate profile along the injection well-depth direction can be obtained by the well logging techniques being widely used in petroleum industry, such as pulsed-neutron tool[47], electromagnetic flow-meter[48] and isotope tracer tool[49]. Based on the well-logging information, we propose an equivalent approach to calculate the reservoir heterogeneity of real EGS, namely:

$$
\delta(y)=\frac{Q(y)-Q(y+\Delta y)}{\Delta y}=\frac{d Q(y)}{d y}
$$

where, $\delta(y)$ denotes the fluid injection flux per unit length of the reservoir depth $y$; $Q(y)$ is the fluid mass flux at the injection well for a depth position, $y$.

Eq. (18) is complemented by Figure 14 for better understanding of the underlying concept. 


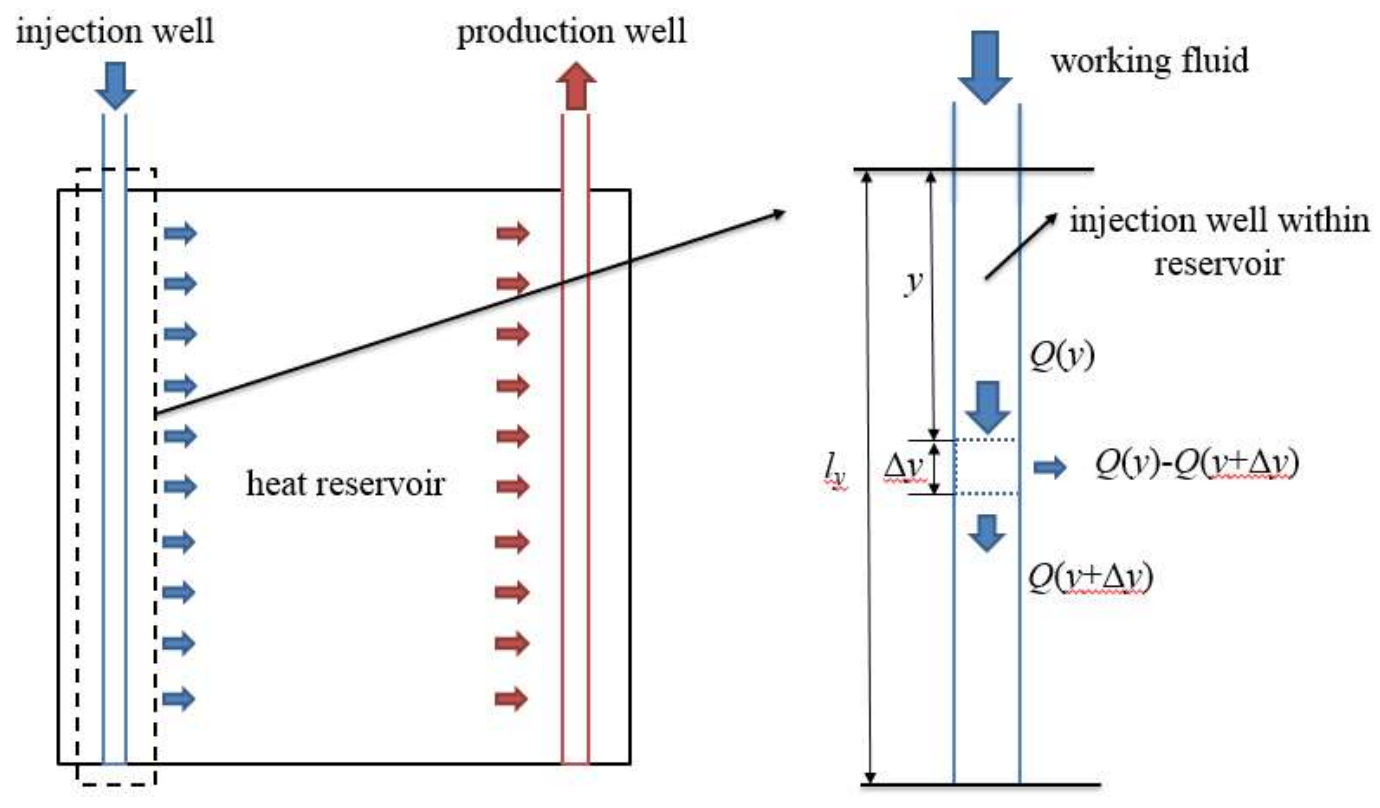

Figure 14 Illustration of the injection fluid flux per unit length along the reservoir depth direction.

For layered heterogeneous reservoirs, $\delta(y)$ is nearly proportional to the permeability at the reservoir depth position, $y$.

$$
\frac{\delta(y)}{\delta_{\text {ave }}}=\frac{k(y)}{k_{\text {ave }}}
$$

where, $\delta_{\text {ave }}$ is the injection flux per unit length of the reservoir depth in a homogeneous reservoir, which is equal to $\left(Q / l_{\mathrm{y}}\right) ; l_{\mathrm{y}}$ is the height of EGS reservoir along its depth direction. Hence, the heterogeneity can be transformed as

$$
\alpha=\frac{1}{\sqrt[n]{\int_{0}^{1}\left[\frac{\delta(y)}{\delta_{\text {ave }}}\right]^{n+1} d\left(\frac{y}{l_{y}}\right)}}
$$

In the previous sections 3 and 4 of the present work, to simplify the heterogeneity in the EGS reservoir, the heterogeneous reservoir samples were 
assumed to be layer-structured, so that the crossflow between the horizontal flow layers is ignored. However, real EGS reservoirs, most likely, do not meet this assumption; therefore, to test the performance of the prediction method when being applied to more practical heterogeneous reservoir cases, $203 \mathrm{D}$ stochastic heterogeneous reservoir cases (i.e. cases 75-94 listed in Table 2) are generated in which the porosity in the reservoir is completely randomly assigned. The location-dependent porosity in the reservoir takes value within the range of 0.008-0.012 while the mean value keeps at 0.01 for all the cases; the permeability calculated by Eq. (5) varies within $10^{-13}$ to $10^{-15} \mathrm{~m}^{2}$.

Horizontal flow path is the shortest fluid flow path from the injection well to the production well. If vertical crossflow becomes strong in the EGS reservoir, the fluid would tend to take a more meandering path through the reservoir, which will extend the fluid residence time. As it is shown in Figure 15, the $\eta$-s for S3DHeR cases are much higher than the $\alpha$ values. If we assign the vertical permeability to 0 (i.e. cases 95-114 listed in Table 2) to extinguish vertical crossflow, the value of $\eta$ obviously drops and it becomes close to the prediction value. This indicates that vertical crossflow is the main cause for the increase in $\eta$. 


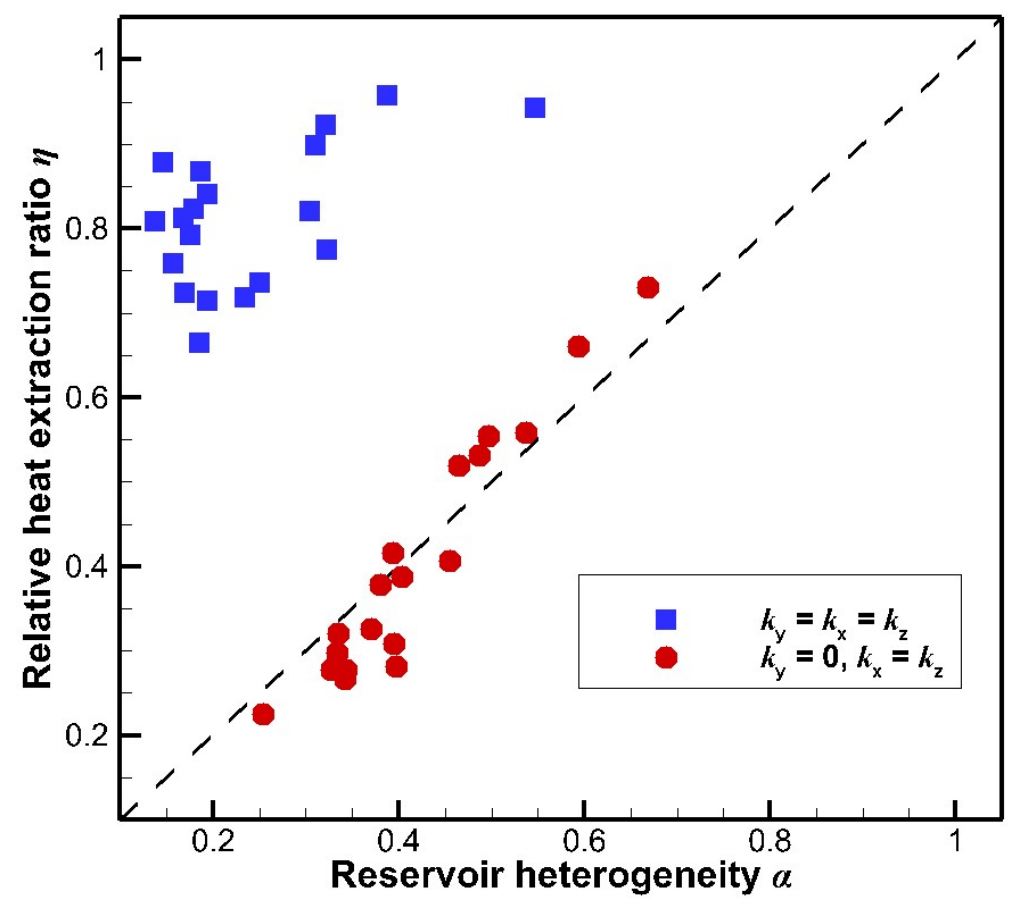

Figure 15 Predicted values compared to simulation results from S3DHeR cases with different anisotropy $(\theta=0.1)$.

Generally, for the actual EGS reservoir which cannot completely meet the layered structure assumption, the heat extraction performance could be higher than the prediction value which is based on reservoir heterogeneity calculated from the well flux information. The primary cause of this underestimate is the disregard of crossflow between the horizontal flow layers. Even though, this prediction is appropriate to be taken as a conservative estimate on the heat performance of an actual EGS reservoir. 


\section{Conclusions}

The effects of the reservoir heterogeneity on EGS heat extraction performance were studied through the simulation of an extensive number of cases. Results indicate that the heat extraction performance is significantly affected by the reservoir heterogeneity and the uneven seepage flow is the primary cause of EGS performance decline.

Based on the layered heterogeneous reservoir model, an approach to quantify the effects of reservoir heterogeneity on EGS heat extraction performance is proposed. The approach encompasses two steps: first, it calculates the reservoir heterogeneity using the measured data on an actual reservoir (e.g. well flux information); then, as a function of the value of the reservoir heterogeneity, the heat that is available from the actual reservoir is determined based on the heat available for an EGS case with homogeneous porous reservoir of comparable dimensions and at the same operating conditions.

The reservoir heterogeneity $\alpha$ contains an adjustment index $n$ to adjust its sensitivity to the preferential flow in reservoir. Based on the simulation results, the optimal adjustment index is correlated with the dimensionless production temperature. In general, the optimal adjustment index tends to be small when the production temperature decline, meanwhile the prediction error increases. This indicates the preferential flow have a significant influence on reservoir heat extraction performance in the early stage of the heat-mining process, and become less influencing as the 
heat-mining process goes on.

According to the simulation results for layered reservoirs, the method, in which the well flux information is employ to calculate reservoir heterogeneity, performs well when the production temperature is within $15 \%$ of the maximum drop; for higher values of the dimensionless production temperature, inter-layer thermal conduction in the reservoir is not irrelevant any more and it should be considered in the formulation. Moreover, for reservoirs of thinner layer thickness, the inter-layer thermal conduction shows more pronounced effect and may come into play for lower values of the dimensionless production temperature at an earlier time point into the EGS heat extraction operation.

When the reservoir is not layer-structured, e.g. real heat reservoirs of $3 \mathrm{D}$ heterogeneity, the method employing the well flux information tends to underestimate the heat extraction performance primarily due to the presence of vertical crossflow in the reservoir. More pertinent approach can better quantify the reservoir heterogeneity is required; an approach based on pulsed injection tracer test [50-52] is in development and may be reported in a future publication.

\section{Acknowledgments}

Financial support received from the China National Science Foundation and Guangdong-Province Joint Project (U1401232), the Key Scientific Development Project of Guangdong Province (2014A030308001), the CAS “100 talents” Program 
(FJ), and the China National Science Foundation (51406213) is gratefully acknowledged. 
Nomenclature

\begin{tabular}{|c|c|c|c|}
\hline$c_{p}$ & heat capacity $(\mathrm{J} / \mathrm{kg} / \mathrm{K})$ & \multicolumn{2}{|c|}{ Greek symbols } \\
\hline$h a$ & $\begin{array}{l}\text { volumetric heat transfer coefficient } \\
\left(\mathrm{W} / \mathrm{m}^{3} / \mathrm{K}\right)\end{array}$ & $\alpha$ & reservoir heterogneity \\
\hline$P$ & pressure $(\mathrm{Pa})$ & $\alpha_{1}$ & reservoir heterogneity (form 1) \\
\hline$k$ & permeability $\left(\mathrm{m}^{2}\right)$ & $\alpha_{2}$ & reservoir heterogneity (form 2) \\
\hline$k_{0}$ & initial permeability $\left(\mathrm{m}^{2}\right)$ & $\varepsilon$ & porosity \\
\hline$k_{\mathrm{ave}}$ & volume average permeability $\quad\left(\mathrm{m}^{2}\right)$ & $\varepsilon_{0}$ & initial porosity \\
\hline$k_{\max }$ & maximum permeability $\left(\mathrm{m}^{2}\right)$ & $\rho$ & density $\left(\mathrm{kg} / \mathrm{m}^{3}\right)$ \\
\hline$n$ & adjustment index & $\mu$ & viscosity $\left(\mathrm{m}^{2} / \mathrm{s}\right)$ \\
\hline$n_{\theta}$ & optimum adjustment index & $\lambda$ & thermal conductivity $(\mathrm{W} / \mathrm{m} / \mathrm{K})$ \\
\hline$T$ & temperature $(\mathrm{K})$ & $\theta$ & dimensionless temperature \\
\hline$T_{\text {in }}$ & injection temperature $(\mathrm{K})$ & $\gamma$ & heat extraction ratio \\
\hline$T_{\text {out }}$ & prodcution temperature $(\mathrm{K})$ & $\gamma_{\mathrm{L}}$ & local heat extraction ratio \\
\hline$t$ & time $(\mathrm{s})$ & $\gamma_{0}$ & heat extraction ratio of HoR \\
\hline$t_{\mathrm{r}, \min }$ & shortest residence time of fluid (s) & $\eta$ & relative heat extraction ratio \\
\hline$u$ & velocity vector $(\mathrm{m} / \mathrm{s})$ & $\delta_{\mathrm{y}}$ & $\begin{array}{l}\text { fluid injection flux per unit length } \\
\text { of the reservoir depth } y(\mathrm{~kg} / \mathrm{s} / \mathrm{m})\end{array}$ \\
\hline$V$ & reservoir volume $\left(\mathrm{m}^{3}\right)$ & $\delta_{0}$ & $\begin{array}{l}\text { fluid injection flux per unit length } \\
\text { in homogeneous reservoir }(\mathrm{kg} / \mathrm{s} / \mathrm{m})\end{array}$ \\
\hline$Q$ & mass flow rate of fluid $(\mathrm{kg} / \mathrm{s})$ & & \\
\hline$Q_{v}$ & volumetric flow rate of fluid $(\mathrm{kg} / \mathrm{s})$ & $\mathrm{Su}$ & uperscripts \\
\hline$q_{\text {well }}$ & $\begin{array}{l}\text { fluid mass flow rate through the } \\
\text { cross section of injection well }(\mathrm{kg} / \mathrm{s})\end{array}$ & $\mathrm{e}$ & effective \\
\hline$l_{\mathrm{x}}$ & length of reservoir (m) & $\mathrm{s}$ & solid or rock \\
\hline$l_{\mathrm{y}}$ & height of reservoir (m) & $\mathrm{f}$ & fluid \\
\hline$l_{\mathrm{z}}$ & width of reservoir (m) & & \\
\hline
\end{tabular}




\section{References}

[1] J. Twidell, T. Weir, Renewable energy resources, in, Routledge, 2015.

[2] J. Goldemberg, World Energy Assessment: Energy and the challenge of sustainability, in, United Nations Pubns, 2000, pp. 165.

[3] I.E. Agency, Key world energy statistics 2015, in, International Energy Agency, 2015, pp. 6.

[4] M. Lacirignola, I. Blanc, Environmental analysis of practical design options for enhanced geothermal systems (EGS) through life-cycle assessment, Renewable Energy, 50 (2013) 901-914.

[5] G.E. Association, 2016 Annual US \& Global Geothermal Power Production Report, Geothermal Energy Association, 2016.

[6] D.W. Brown, D.V. Duchane, G. Heiken, V.T. Hriscu, Mining the earth's heat: hot dry rock geothermal energy, Springer Science \& Business Media, 2012.

[7] K. Breede, K. Dzebisashvili, X. Liu, G. Falcone, A systematic review of enhanced (or engineered) geothermal systems: past, present and future, Geothermal Energy, 1(1) (2013) 1-27.

[8] J.W. Tester, B.J. Anderson, A. Batchelor, D. Blackwell, R. DiPippo, E. Drake, J. Garnish, B. Livesay, M. Moore, K. Nichols, The future of geothermal energy, 2006.

[9] R. DiPippo, Geothermal power plants: principles, applications, case studies and environmental impact, Butterworth-Heinemann, 2012.

[10] E. Robinson, R. Potter, B. McInteer, J. Rowley, D. Armstrong, R. Mills, Preliminary study of the nuclear subterrene, Los Alamos Scientific Lab., N. Mex., 1971.

[11] B.R. Lawn, E. Fuller, Equilibrium penny-like cracks in indentation fracture, Journal of Materials Science, 10(12) (1975) 2016-2024.

[12] C. Raleigh, P. Witherspoon, A. Gringarten, Y. Ohnishi, Multiple hydraulic fracturing for the recovery of geothermal energy, EOS, Trans., Am. Geophys. Union;(United States), 55(4) (1974).

[13] R. Baria, J. Baumgärtner, F. Rummel, R.J. Pine, Y. Sato, HDR/HWR reservoirs: concepts, understanding and creation, Geothermics, 28(4) (1999) 533-552.

[14] R. Pine, A. Batchelor, Downward migration of shearing in jointed rock during hydraulic injections, in: International Journal of Rock Mechanics and Mining Sciences \& Geomechanics Abstracts, Elsevier, 1984, pp. 249-263.

[15] T.L. Blanton, An experimental study of interaction between hydraulically induced and pre-existing fractures, in: SPE Unconventional Gas Recovery Symposium, Society of Petroleum Engineers, 1982.

[16] A.S. Batchelor, Reservoir behaviour in a stimulated hot dry rock system, in: 11th Workshop on Geothermal Reservoir Engineering, Stanford University Calif, 1986.

[17] G.G. Tsypkin, C. Calore, M. Marcolini, Mathematical modeling of cold water injection into a depleted high-temperature geothermal reservoir, High Temperature, 44(3) (2006) 450-457.

[18] D.W. Brown, Hot dry rock geothermal energy: important lessons from Fenton Hill, in: Proceedings of the Thirty-Fourth Workshop on Geothermal Reservoir Engineering, Stanford University, CA, 2009.

[19] J. Willis-Richards, T. Wallroth, Approaches to the modelling of HDR reservoirs: a review, Geothermics, 24(3) (1995) 307-332.

[20] A. Gringarten, J. Sauty, A theoretical study of heat extraction from aquifers with uniform regional flow, Journal of Geophysical Research, 80(35) (1975) 4956-4962.

[21] A. Gringarten, P. Witherspoon, Y. Ohnishi, Theory of heat extraction from fractured hot dry 
rock, Journal of Geophysical Research, 80(8) (1975) 1120-1124.

[22] R. Schulz, Analytical model-calculations for heat-exchange in a confined aquifer, JOURNAL OF GEOPHYSICS-ZEITSCHRIFT FUR GEOPHYSIK, 61(1) (1987) 12-20.

[23] N. Heuer, T. Küpper, D. Windelberg, Mathematical model of a hot dry rock system, Geophysical Journal International, 105(3) (1991) 659-664.

[24] S.K. Sanyal, S.J. Butler, An analysis of power generation prospects from enhanced geothermal systems, Geothermal Resources Council Transactions, 29 (2005).

[25] M.J. O'Sullivan, K. Pruess, M.J. Lippmann, State of the art of geothermal reservoir simulation, Geothermics, 30(4) (2001) 395-429.

[26] J. Long, J. Remer, C. Wilson, P. Witherspoon, Porous media equivalents for networks of discontinuous fractures, Water Resources Research, 18(3) (1982) 645-658.

[27] P.A. Witherspoon, J.S. Wang, K. Iwai, J.E. Gale, Validity of cubic law for fluid flow in a deformable rock fracture, Water Resources Research, 16(6) (1980) 1016-1024.

[28] E. Kalinina, T. Hadgu, S. McKenna, T. Lowry, Bridging the gap between complex numerical modeling and rapid scenario assessment: a dimensionless parameter approach for enhanced geothermal systems, Trans Geoth Resour Counc, 35 (2011) 407-412.

[29] S. Nakanishi, Y. Kawano, N. Todaka, C. Akasaka, M. Yoshida, N. Iwai, A reservoir simulation of the Oguni field, Japan, using MINC type fracture model, in: Proc. World Geothermal Congress, 1995, pp. 1721-1726.

[30] M. Grant, Geothermal reservoir engineering, Elsevier, 2013.

[31] S.K. Garg, J. Combs, Appropriate use of USGS volumetric "heat in place" method and Monte Carlo calculations, in: Proceedings 34th Workshop on Geothermal Reservoir Engineering, Stanford university, Stanford, California, USA, 2010.

[32] J. Chen, F. Jiang, Designing multi-well layout for enhanced geothermal system to better exploit hot dry rock geothermal energy, Renewable Energy, 74 (2015) 37-48.

[33] C.F. Williams, Updated methods for estimating recovery factors for geothermal resources, in: Proceedings, Thirty-Second Workshop on Geothermal Reservoir Engineering. Stanford University, 2007.

[34] J. Chen, F. Jiang, A numerical study of EGS heat extraction process based on a thermal non-equilibrium model for heat transfer in subsurface porous heat reservoir, Heat and Mass Transfer, (2015) 1-13.

[35] C. Vogt, G. Marquart, C. Kosack, A. Wolf, C. Clauser, Estimating the permeability distribution and its uncertainty at the EGS demonstration reservoir Soultz-sous-Forets using the ensemble Kalman filter, Water Resources Research, 48 (2012).

[36] E.A. Kalinina, K.A. Klise, S.A. McKenna, T. Hadgu, T.S. Lowry, Applications of fractured continuum model to enhanced geothermal system heat extraction problems, SpringerPlus, 3(1) (2014) $1-13$.

[37] A.R. Shaik, S.S. Rahman, N.H. Tran, T. Thanh, Numerical simulation of Fluid-Rock coupling heat transfer in naturally fractured geothermal system, Applied Thermal Engineering, 31(10) (2011) 1600-1606.

[38] E.M. Llanos, S.J. Zarrouk, R.A. Hogarth, Numerical model of the Habanero geothermal reservoir, Australia, Geothermics, 53 (2015) 308-319.

[39] C. Xu, P.A. Dowd, Z.F. Tian, A simplified coupled hydro-thermal model for enhanced geothermal systems, Applied Energy, 140 (2015) 135-145. 
[40] C. Vogt, N. Klitzsch, V. Rath, On self-potential data for estimating permeability in enhanced geothermal systems, Geothermics, 51 (2014) 201-213.

[41] N. Doonechaly, S. Rahman, A. Kotooussov, A realistic assessment of recoverable thermal energy from Australian geothermal reservoirs: a simulation study, in: Australian Geothermal Energy Conference (5th: 2012: Sydney, Australia), 2012.

[42] F. Jiang, L. Luo, J. Chen, A novel three-dimensional transient model for subsurface heat exchange in enhanced geothermal systems, International Communications in Heat and Mass Transfer, 41 (2013) 57-62.

[43] F. Jiang, J. Chen, W. Huang, L. Luo, A three-dimensional transient model for EGS subsurface thermo-hydraulic process, Energy, 72 (2014) 300-310.

[44] W. Cao, W. Huang, F. Jiang, Numerical study on variable thermophysical properties of heat transfer fluid affecting EGS heat extraction, International Journal of Heat and Mass Transfer, 92 (2016) 1205-1217.

[45] T. Xu, E. Sonnenthal, N. Spycher, K. Pruess, TOUGHREACT - A simulation program for non-isothermal multiphase reactive geochemical transport in variably saturated geologic media: Applications to geothermal injectivity and $\mathrm{CO} 2$ geological sequestration, Computers \& Geosciences, 32(2) (2006) 145-165.

[46] M.A. Grant, S.K. Garg, Recovery factor for EGS, in: Proceedings of the 37th Workshop on Geothermal Reservoir Engineering, Stanford University, Stanford, California, USA 2012.

[47] B.A. Roscoe, C. Lenn, Oil and Water Flow Rate Logging in Horizontal Wells Using Chemical Markers and a Pulsed-Neutron Tool, in: Abu Dhabi International Petroleum Exhibition and Conference, Society of Petroleum Engineers, 1996.

[48] L. Dianlong, W. Yunfei, W. Wang, Application of the electromagnetic flowmeter to polymer injection profile logging [J], Petroleum Instruments, 3 (2001) 012.

[49] L. Yingtai, X. Aifang, Y. Meilan, Q. Yubao, W. Yunhong, Y. Qingxiu, Overview of the injection well flow rate log instruments [J], Petroleum Instruments, 4 (2005) 001.

[50] B. Sanjuan, J.-L. Pinault, P. Rose, A. Gérard, M. Brach, G. Braibant, C. Crouzet, J.-C. Foucher, A. Gautier, S. Touzelet, Tracer testing of the geothermal heat exchanger at Soultz-sous-Forêts (France) between 2000 and 2005, Geothermics, 35(5) (2006) 622-653.

[51] P.E. Rose, S.D. Johnson, P. Kilbourn, C. Kasteler, Tracer testing at Dixie Valley, Nevada using 1-naphthalene sulfonate and 2, 6-naphthalene disulfonate, in: Proceedings, Twenty-Seventh Workshop on Geothermal Reservoir Engineering, Stanford University, Stanford, CA, January, 2002, pp. 28-30.

[52] B.F. Ayling, R.A. Hogarth, P.E. Rose, Tracer testing at the Habanero EGS site, central Australia, Geothermics, 63 (2015) 15-26. 